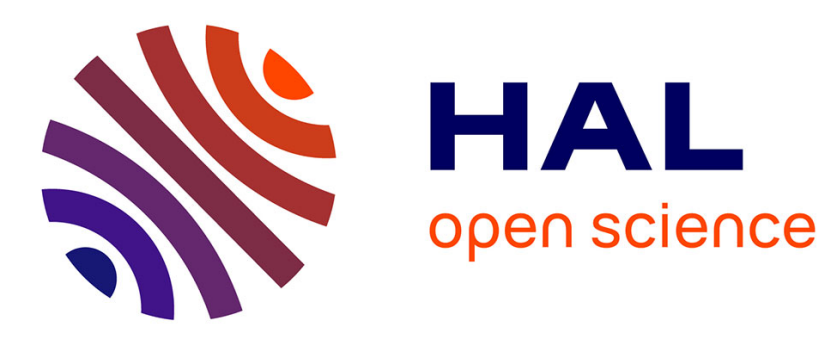

\title{
Non-local Active Contours
}

\author{
Jung Miyoun, Gabriel Peyré, Laurent D. Cohen
}

\section{To cite this version:}

Jung Miyoun, Gabriel Peyré, Laurent D. Cohen. Non-local Active Contours. SSVM'11: 3rd International Conference on Scale Space and Variational Methods in Computer Vision, May 2011, Ein-Gedi, Israel. hal-00591281

\section{HAL Id: hal-00591281 https://hal.science/hal-00591281}

Submitted on 8 May 2011

HAL is a multi-disciplinary open access archive for the deposit and dissemination of scientific research documents, whether they are published or not. The documents may come from teaching and research institutions in France or abroad, or from public or private research centers.
L'archive ouverte pluridisciplinaire HAL, est destinée au dépôt et à la diffusion de documents scientifiques de niveau recherche, publiés ou non, émanant des établissements d'enseignement et de recherche français ou étrangers, des laboratoires publics ou privés. 


\title{
Non-local Active Contours
}

\author{
Miyoun Jung, Gabriel Peyré and Laurent D. Cohen \\ Ceremade, Université Paris-Dauphine, 75775 Paris Cedex 16, France \\ \{jung, peyre, cohen\}@ceremade.dauphine.fr
}

\begin{abstract}
This article introduces a new image segmentation method that makes use of non-local comparisons between pairs of patches of features. A non-local energy is defined by summing the interactions between pairs of patches inside and outside the segmented domain. A maximum radius of interaction can be adapted to fit the amount of variation of the features inside and outside the region to segment. This non-local energy is minimized using a level set approach. The corresponding curve evolution defines a non-local active contour that converges to a local minimum of our energy. In contrast to previous segmentation methods, this approach only requires a local homogeneity of the features inside and outside the region to segment. This does not impose a global homogeneity as required by region-based segmentation methods. This comparison principle is also less sensitive to initialization than edge-based approaches. We instantiate this novel framework using patches of intensity or color values as well as Gabor features. This allows us to segment regions with smoothly varying intensity or colors as well as well as complicated textures with a spatially varying local orientation.
\end{abstract}

\section{Introduction}

Image segmentation refers to the process of partitioning an image into several regions or locating objects and boundaries. This paper considers a variational minimization problem for segmentation, which aims to find a contour representing the boundary of objects, by minimizing an energy functional composed of a contour smoothing term and an attraction term that pulls the contour towards the object boundaries. The curve (locally) minimizing the energy functional, located at the object boundaries, is obtained by curve evolution or active contours: starting with a given initial curve and evolving it to the correct steady state, the object boundaries. Active contours have been represented either by explicit parametric representation [1] or by the implicit level set representation of [2]. The level set representation has widely been used because it allows automatic topology changes of the contour such as merging and breaking, and the computations are made on a fixed rectangular grid. Many existing active contour models segment an image according to edge information and/or region information.

Edge-based approaches. Edge-based active contour models use edge detection functions depending on the image gradient and evolve contours towards sharp 
gradients of pixel intensity. The first work was the snakes model by Kass et al. [1]. Then, many edge-based active contour models such as balloon [3], geometric [4], [5], [6] models were proposed. In particular, Caselles et al. [6] proposed an intrinsic geometric model, geodesic active contours, where the curve evolution is handled by the level set method [2] proposed by Osher and Sethian. In this model, the evolving curve moves by mean curvature, but with an extra factor in the speed, by the stopping edge-function. Therefore, the curve stops on the edges, where the edge-function vanishes. Although these classical snakes or geometric active contour models are quite effective, they are usually not robust to noise because noise also has large gradients. These models need in addition to perform a-priori smoothing, to smooth out the noise. This can therefore produce a not very accurate location of edges.

Region-based approaches. Region-based active contour models incorporate region information so that image within each segmented region has a homogeneous characteristics, such as intensities and textures. A region-based energy for an active contour was proposed in [7]. This was a reduced form of the Mumford-Shah functional [8] where the image was approximated by a piecewise smooth function inside objects and a smooth background. Chan and Vese [9] proposed an active contours without edges model, which is also based on techniques of curve evolution and level set methods, but the gradient-based information is replaced by a criterion related to region homogeneity. This model approximates an image by a two-phase piecewise constant function. The active contours without edge model was also extended to vector valued images [10] and to texture segmentation [11].

Kimmel [12] proposed a hybrid model by incorporating a more general weighted arc-length in the active contours without edges model. Sagiv et al. [13] applied the integrated approach, by incorporating multi-channel approaches [10], [11], to the problem of texture segmentation.

In this article, we propose an active contour model with a novel energy functional using pairwise interaction of features inside and outside the object, which allows to only constrain the local homogeneity, in contrast to the Chan-Vese approach. The local homogeneity property allows our model to capture regions with features that vary spatially in a smooth way, as well as to segment several separated objects with different features.

Remark that, a class of models [14], [15], [16] in the framework of MumfordShah or Chan-Vese models has been proposed to perform the segmentation of images with intensity inhomogeneity. These also utilize region-based techniques and incorporate the benefits of local information. The difference is that our model is developed in the non-local framework using patches, illustrated in the following paragraphs.

Image features. In this work, we consider different image features based on given images. The choice of features is difficult and critical to get an optimal segmentation result. For a scalar image, the gray-level value or intensity can be enough to characterize each pixel. If the image is composed of multiple channels (such as color images), then each pixel is described by a vector of intensities. For texture 
images, the pixel intensity value does not give pertinent information. A very popular class of texture features are the filter-based features of the given image. For instance, Gabor filter has often been implemented in texture segmentation [17], [11], [13] because it can segment images having region differences in spatial frequency, density of elements, orientation and phase. In particular, Sandberg et. al [11] incorporated the multiple Gabor transforms, obtained by convolving the Gabor functions with the original textured image, with the vector valued active contours without edges algorithm [10]. A recent promising image feature to represent and process textures is the image intensity patch around the current pixel. The information on a close neighborhood around the current pixel is extracted and leads to semi-local information at each pixel. The patch idea as feature vector was first introduced for texture synthesis [18], [19], then for image denoising, illustrated in the following paragraph.

We incorporate the patch idea with selected image features: for instance, for texture images, we use Gabor transforms as image features, and we consider the non-local interaction between pairs of patches of the features.

Non-local image processing. Nonlocal methods in image processing have been explored in many papers because they are well adapted to texture denoising. Buades et al [20] proposed to compute the weight matrix with patch differences and denoise the image with a non-local averaging, which is the well-known nonlocal means filter. Kinderman et al. [21], Gilboa and Osher [22], and Peyré et al. [23] proposed non-local energy functionals, and these functionals were used to solve various image processing problems such as denoising, inpainting, superresolution and compressive sensing. The idea of functionals on nonlocal-graphs, in a regularization process, has also been used for image segmentation in a semisupervised [24], [25], [26] or an unsupervised [27], [28] settings.

In our work, we use a non-local energy that enforces the similarity of features both located either inside or outside the object. Using a level set formulation, this defines an attraction term pulling the contour towards the object boundaries. This is contrast to the existing non-local based segmentation methods that use non-local energy terms only as regularization terms. Note that our work is related to some developments that start from the work of Shi and Malik [29].

Contributions. This article introduces a novel non-local energy for image/texture segmentation. In contrast to existing energy, we use pairwise interaction of features, which allows to only constrain the local homogeneity. This local homogeneity is crucial to capture regions with smoothly spatially varying features, such as color gradient or oriented textures. This is also useful to segment several separated objects with different features.

\section{Non-local Active Contours}

The goal is to segment an image $f:[0,1]^{2} \rightarrow \mathbb{R}^{d}$, where $d$ is dimensionality of the feature space. For instance one might consider $d=1$ for gray-valued images, 
$d=3$ for color images. To segment a texture image, $f(x)$ is computed as a high dimensional vectors which is the output of a directional filter bank.

Since we aim at proposing a generic segmentation framework, we do not specify the exact nature of the features in this section. Sections 3.2 and 3.3 detail some typical examples of features spaces.

\subsection{Pairwise Patch Interaction}

To be able to be less sensitive to noise in the image, we consider patches of features around each pixel $x \in[0,1]^{2}$ :

$$
\forall t \in[-\tau / 2, \tau / 2]^{2}, \quad p_{x}(t)=f(x+t) .
$$

Patch-based processing of images has been used extensively for a very long time in stereo and image matching in general, and has been very popular since the introduction of the non-local means denoising method.

Similarly to non-local denoising, we consider the non-local interaction between pairs of patches, measured using a weighted $L^{2}$ distance

$$
d\left(p_{x}, p_{y}\right)=\int_{t} G_{a}(t)\left\|p_{x}(t)-p_{y}(t)\right\|^{2} \mathrm{~d} t \quad \text { where } \quad G_{a}(t)=e^{-\frac{\|t\|^{2}}{2 a^{2}}} .
$$

The Gaussian weight is used to give more influence to the central pixel, but does not play a major role in our method.

\subsection{Pairwise Interaction Energy}

In its simplest form, the segmentation problem corresponds to the computation of some region $\Omega \subset[0,1]^{2}$ that should capture the objects of interest. This is usually performed in some variational framework where $\Omega$ solves an optimization problem.

The local homogeneity of the region (and of its complementary) is measured by considering all possible pairwise patch interaction at a given scale $\sigma>0$. This gives rise to the following pairwise interaction energy of a region

$$
E(\Omega)=\iint_{\Omega \times \Omega} G_{\sigma}(x-y) d\left(p_{x}, p_{y}\right) \mathrm{d} x \mathrm{~d} y+\iint_{\Omega^{c} \times \Omega^{c}} G_{\sigma}(x-y) d\left(p_{x}, p_{y}\right) \mathrm{d} x \mathrm{~d} y .
$$

where $\Omega^{c}=[0,1]^{2} \backslash \Omega$ is the complementary of the region.

The parameter $\sigma>0$ is important since it controls the scale of the local homogeneity one requires for the segmented object. If the region is made of a nearly constant pattern, one should use a large $\sigma$. In contrast, if the region exhibits fast feature variations, $\sigma$ should be chosen smaller. For simplicity, we use the same scale for both inside and outside the region, but one could of course use two distinct parameters. 


\subsection{Non-local Active Contour Energy}

In order to perform the segmentation, we use a level set framework [2] where one computes a function $\varphi:[0,1] \rightarrow \mathbb{R}$ so that $\Omega=\{x \backslash \varphi(x)>0\}$.

The integration inside and outside the domain is carried over using a smoothed Heaviside function

$$
H(x)=\frac{1}{2}+\frac{1}{\pi} \operatorname{atan}(x / \varepsilon) .
$$

The parameter $\varepsilon$ should be chosen small enough to obtain a sharp region boundary, but not too small to avoid numerical instabilities. In the numerical examples, we use $\varepsilon=1 / n$ for a discretized image of $n \times n$ pixels.

The energy (1) on regions is turned into an energy on the level set function $\varphi$, enforcing the similarity of features located inside and outside $\Omega$,

$$
E(\varphi)=\iint \rho(H(\varphi(x)), H(\varphi(y))) G_{\sigma}(x-y) d\left(p_{x}, p_{y}\right) \mathrm{d} x \mathrm{~d} y
$$

where $\rho$ is an indicator function such that $\rho(u, v)=1$ if $u=v, 0$ otherwise. In practice, we used $\rho(u, v)=1-|u-v|$. The meaning of this term is a way to consider only pairs of points for which $\varphi$ has the same sign. Note that other binary interaction function $\rho$ could be used as well, such as $\rho(u, v)=u v+(1-$ $u)(1-v)$ (when $u=H(\varphi(x)), v=H(\varphi(y)))$ and $\rho(u, v)=1-|u-v|^{2}$.

To enforce the regularity of the extracted region, following previous works in active contours, we penalize the length of the boundary, which is computed as

$$
L(\varphi)=\int\|\nabla H(\varphi(x))\| \mathrm{d} x=\int H^{\prime}(\varphi(x))\|\nabla \varphi(x)\| \mathrm{d} x
$$

where $\nabla H(\varphi(x))$ is the gradient at point $x$ of the function $H(\varphi)$.

Our non-local active contour method compute the segmentation as a stationary point of the energy

$$
\min _{\varphi} E(\varphi)+\gamma L(\varphi)
$$

where $\gamma>0$ is a parameter that should be adapted to the expected regularity of the boundary of the region.

Using the gradient descent with an artificial time $t \geqslant 0$ leads to the evolution equation for $\varphi$ :

$$
\frac{\partial \varphi}{\partial t}=-(\nabla E(\varphi)+\gamma \nabla L(\varphi))
$$

where the gradients are computes as

$$
\begin{gathered}
\nabla E(\varphi)(x)=\int\left(\partial_{1} \rho\right)(H(\varphi(x)), H(\varphi(y))) G_{\sigma}(x-y) d\left(p_{x}, p_{y}\right) \mathrm{d} y H^{\prime}(\varphi(x)), \\
\nabla L(\varphi)(x)=-\operatorname{div}\left(\frac{\nabla \varphi(x)}{\|\nabla \varphi(x)\|}\right) H^{\prime}(\varphi(x)) .
\end{gathered}
$$


Numerical implementation details. The segmentation is applied to a discretized image $f$ of $n \times n$ pixels. The length energy (2) is computed using a finite difference approximation of the gradient.

The algorithm consists of two steps: given an image $f$, the weight function $w(x, y)=G_{\sigma}(x-y) d\left(p_{x}, p_{y}\right)$ is constructed based on the selected features, and then the evolution equation (3) for $\varphi$ is solved with an explicit scheme. Note that $H^{\prime}(\varphi)$ is replaced by $\|\nabla \varphi\|$. To ensure the stability of the level set evolution (3), one needs to re-initialize it from time to time. This corresponds to replacing $\varphi$ by the signed distance function to the level set $\{x \backslash \varphi(x)=0\}$.

The size of the windowing function $G_{\sigma}(x-y)$ depends on the initial curve: if the initial curve is far away from the object boundaries, then a large windowing function may be required. Here, $31 \times 31$ or $41 \times 41$ are used with a fixed $\sigma=10$ for $100 \times 100$ or $200 \times 200$ images. The choice of the size of patch and the parameter $a$ in $G_{a}$ depends on the image features: for instance, for image features depending on intensity, $3 \times 3$ patch with $a=0.5$ is used.

\section{Experimental Results and Comparisons}

This section presents experimental results with synthetic and real images.

\subsection{Hybrid Region/Edge based Active Contours}

We compare our approach with both region-based and edge-based active contour segmentation methods. We compare our method with segmentations obtained by minimizing a hybrid energy of the form

$$
\min _{\varphi, p} \alpha E_{r}(\varphi, p)+(1-\alpha) E_{c}(\varphi)+\gamma L(\varphi)
$$

where $\alpha$ weights the influence of the region term $E_{r}$ and the edge term $E_{c}$ :

$$
\begin{gathered}
E_{r}(\varphi, p)=\lambda_{1} \int H(\varphi(x)) d\left(p_{x}, p_{1}\right) \mathrm{d} x+\lambda_{2} \int(1-H(\varphi(x))) d\left(p_{x}, p_{2}\right) \mathrm{d} x, \\
E_{c}(\varphi)=\mu \int\|\nabla H(\varphi(x))\| g(x) \mathrm{d} x,
\end{gathered}
$$

with positive parameters $\lambda_{1}, \lambda_{2}, \mu$ and a positive edge function $g$, and where $p$ represents the expected constant value of the features inside and outside the object. In particular, we consider the geodesic active contour model $(\alpha=0$, GAC model [6]) with adding balloon force term $\eta g(x)\|\nabla \varphi(x)\|$, the region-based model $(\alpha=1)$ of Chan and Vese [9], and the integrated region/edge based model $(\alpha=1 / 2)$ of Sagiv et al. [13], called IAC model:

$$
\begin{aligned}
& \text { GAC: } \frac{\partial \varphi}{\partial t}=\mu\|\nabla \varphi\| \operatorname{div}\left(g \frac{\varphi}{\|\varphi\|}\right)+\eta g\|\nabla \varphi\|, \\
& \text { Chan-Vese: } \frac{\partial \varphi}{\partial t}=\|\nabla \varphi\|\left\{-\lambda_{1} d\left(p_{x}, p_{1}\right)+\lambda_{2} d\left(p_{x}, p_{2}\right)+\gamma \operatorname{div}\left(\frac{\varphi}{\|\varphi\|}\right)\right\}, \\
& \text { IAC: } \quad \frac{\partial \varphi}{\partial t}=\frac{1}{2}\|\nabla \varphi\|\left\{\mu \operatorname{div}\left(g \frac{\varphi}{\|\varphi\|}\right)-\lambda_{1} d\left(p_{x}, p_{1}\right)+\lambda_{2} d\left(p_{x}, p_{2}\right)\right\},
\end{aligned}
$$



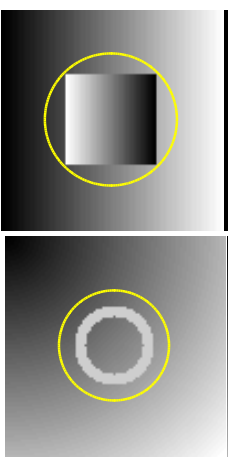

Our model: $k=0$
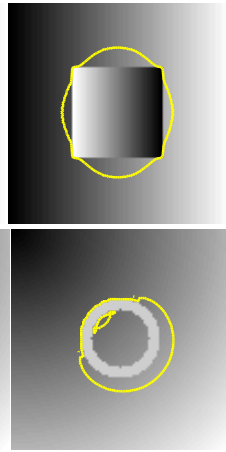

$k=20$
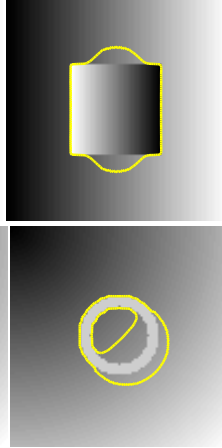

$k=50$
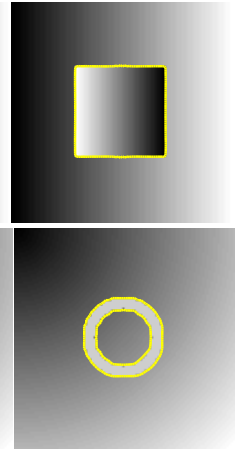

$k=150$
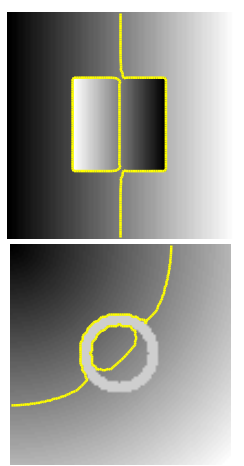

Chan-Vese

Fig. 1. Detection of object with spatially varying background or object, and comparison with Chan-Vese model $(\alpha=1)$ in (4). $100 \times 100$ image and $31 \times 31$ windowing function is used. $k$ is the iteration number.

where $\eta g\|\nabla \varphi\|$ is a balloon force term that helps to avoid poor local minima by forcing moving the curve forward/outward (depending on the sign of $\eta$ ). Note that, in practice, we use an edge function $g(x)=\frac{1}{\delta^{2}+G_{b_{1}} *\left\|\nabla\left(G_{b_{2}} * f\right)(x)\right\|^{p}}$ with $\delta^{2}=0.1$ and $p, b_{1}, b_{2}>0$, and then we normalize it from 0 to 1 . And we let $\lambda_{1}=\lambda_{2}=1$.

\subsection{Gray-level and Color Features}

The simplest features $f(x)$ are the values of the image itself.

In the numerical examples, we use the edge function with $p=1, G_{b_{1}}$ with $b_{1}=0.5$ (or 1 for noisy image) and $G_{b_{2}}=1$.

In Fig. 1 and 2, we test our method on several synthetic images with spatially varying background and/or object, or with several separated objects with different intensities. In all the examples, our model correctly detects the objects. This is due to the local homogeneity property of our model mentioned in Section 2.3, which is contrast to the two-phase Chan-Vese model requiring a global homogeneity in each region. The first example in Fig. 1 well demonstrates the effect of this property. The second example shows in addition the detection of interior contour. In the first example in Fig. 2, the bottom object has spatially varying intensities, and moreover the intensities of its left side are close to the ones of the background. Thus, Chan-Vese model (see Fig. 3) fails to segment this piecewise smooth object, regarding its left side as background, while our model captures the boundary with small gradients. Furthermore, Fig. 2 shows the detection of multiple separated objects with different intensities, unlike two phase Chan-Vese model. Lastly, we note that our model needs small number of iterations (around 150 iterations) to obtain final curves, even with an explicit scheme.

Fig. 3 presents the results of existing edge-based and/or region-based models, given in (4): $\alpha=0$ with balloon force term $\eta g(x)\|\nabla \varphi(x)\|$ (geodesic model), 

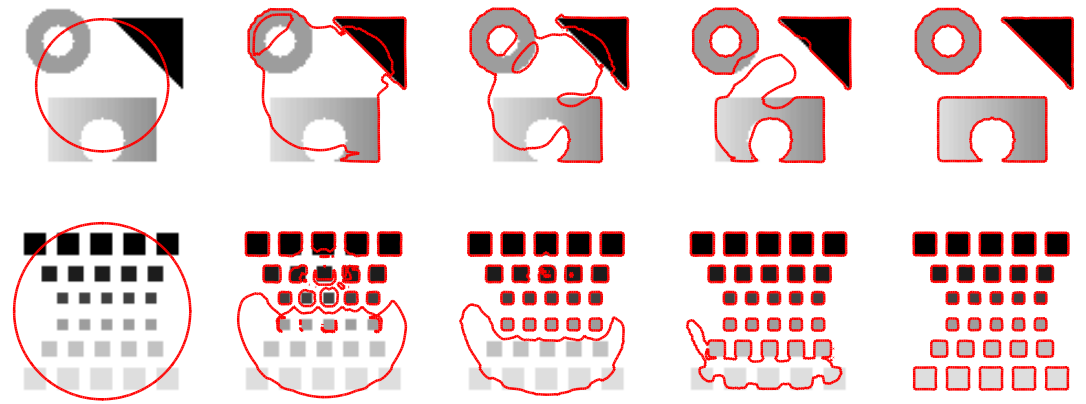

$k=0$

$k=10$

$k=20$

$k=50$

final curve

Fig. 2. Detection of objects with spatially varying object, or with several separated objects with different intensities, or with various shapes, using our model. $k$ is the iteration number, and final curves are obtained at $k=80$ (top) and $k=120$ (bottom).

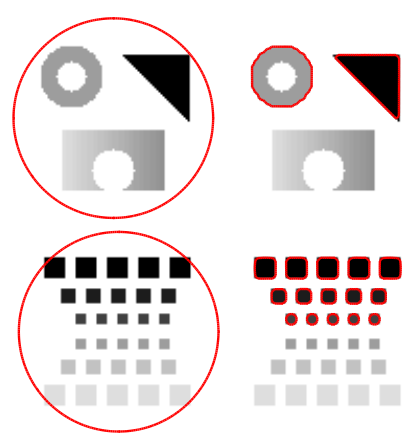

$\alpha=0$ with balloon force
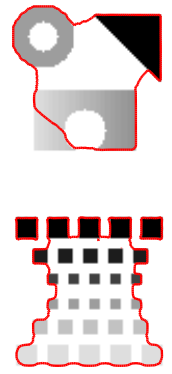
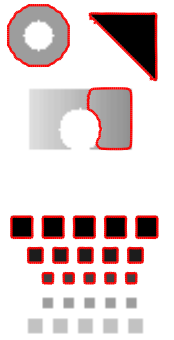

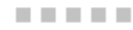

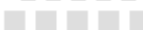

$\alpha=1 / 2$ and two different $\mu$
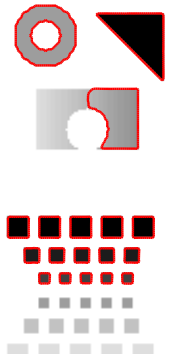

$\alpha=1$

Fig. 3. Final curves of models given in (4): $\alpha=0$ with balloon force term (GAC), $\alpha=1 / 2$ (IAC) with two different but close parameters $\mu, \alpha=1$ (Chan-Vese). GAC: $\mu=1, \eta=-0.3$. IAC: (top) $\mu=3.6$ and 3.5 , (bottom) $\mu=1$ and 0.9 . IAC and Chan-Vese models used initial curves given in Fig. 2.
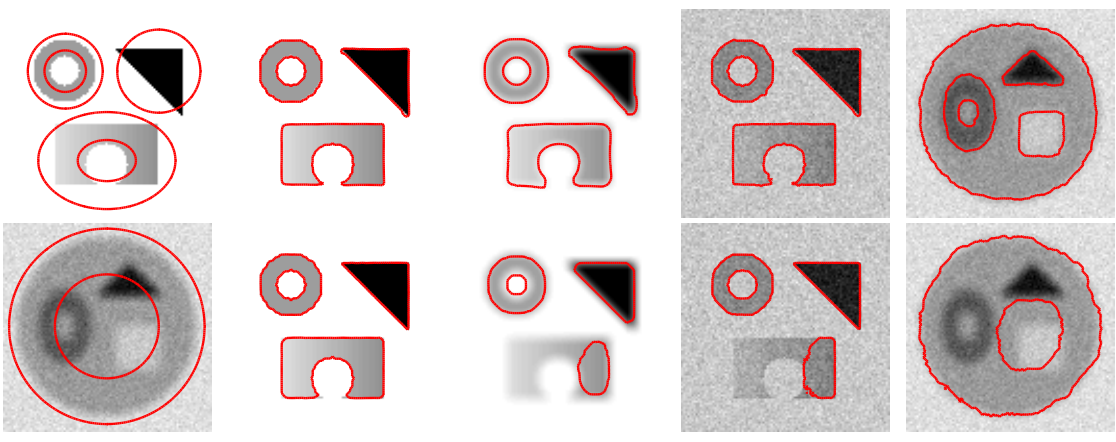

Fig. 4. Detection of objects from blurred and/or noisy images. 1st column: initial curves used, 2nd-5th columns: final curves of our model (Top) and IAC model (Bottom). 
$\alpha=1 / 2$ (integrated active contour model), $\alpha=1$ (region-based Chan-Vese model). For the IAC model, two final curves are shown with two different but close parameters $\mu\left(\mu_{1}>\mu_{2}\right)$. Because $\mu$ is a balancing term between the regionbased and edge-based energies, when $\mu>\mu_{1}$ (or $\mu<\mu_{2}$ ), the model tends to act like the geodesic snake model (or Chan-Vese model). Thus, with the given initial curves, all the models fail to detect the correct object boundaries. Note that, with good initial curves surrounding all the boundaries, IAC model was able to detect the boundaries, as shown in the 2nd column in Fig. 4, with large values of $\mu$, while in our model one circle around objects as an initial curve was enough for segmentation. Thus, our model is less sensitive to the choice of initial curves than edge-based active contour models.

Fig. 4 presents how our model works on noisy images, and detection of objects with blurred boundaries. The 2 nd-4th columns present a clear and clean image (2nd), given in Fig. 2, and a blurred and noisy version of it, respectively. However, IAC model fails to locate boundaries with small gradients in blurred or noisy images, even with good initial curves. These examples show that our model detects object boundaries with small gradients as well as that it is not sensitive to noise unlike edge-based models.

In Fig. 5, we test our method on real color images. We compare our model with the vector-valued Chan-Vese model [10] and IAC model. By using an initial curve near the boundary of object(s) and a small windowing function, our model could detect the boundary of non-homogeneous object(s). The segmentation result is fairly good, comparing with Chan-Vese model and IAC model that only capture part of object(s). On the other hand, these examples also show a limitation of our model: in order to detect the boundary of non-homogeneous objects, the initial curve needs to be located near the object boundary so that a small windowing function can be used.

\subsection{Gabor Features}

To segment a texture image, one can use the energy of the output of a dictionary multi-scale filter bank. Given an image $f_{0}$, one computes each $f(x) \in$ $\mathbb{R}^{d}$ as the magnitude of $d$ complex filters

$$
\forall \ell \in\{0, \ldots, d-1\}, \quad f_{\ell}(x)=\left|f_{0} \star h_{\ell}\right|
$$

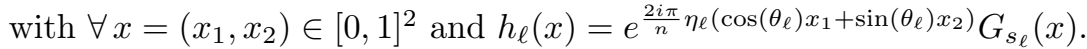

The parameter $\eta_{\ell}>0$ is the frequency of the filtering, $\theta_{\ell} \in[0, \pi)$ is the orientation and $s_{\ell}>0$ is the spacial width of the filter. In the numerical examples, the parameters $\eta_{\ell}, \theta_{\ell}, s_{\ell}$ are fine-tuned to obtain the best texture representation. Note that the energies (4) incorporating Gabor features and multi-channel approach have been used for texture segmentation in [11] (Gabor based multichannel Chan-Vese model) and [13] (IAC model).

Fig. 6 presents our texture segmentation result and comparison with Gabor based Chan-Vese model [11]. In this case, we use $d=8$ filters with $\eta_{\ell} \in$ 


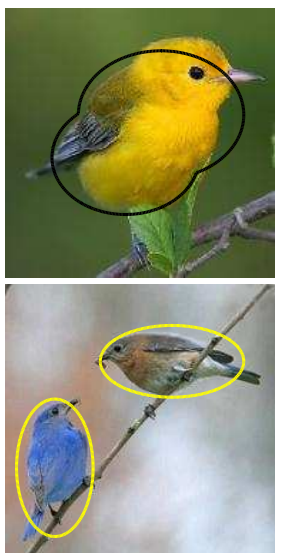

initial curve
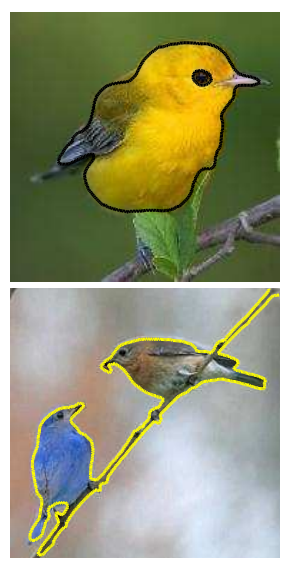

our model
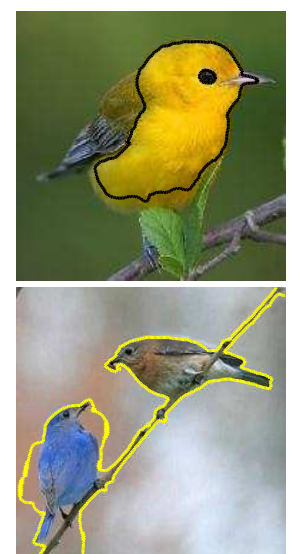

Chan-Vese model

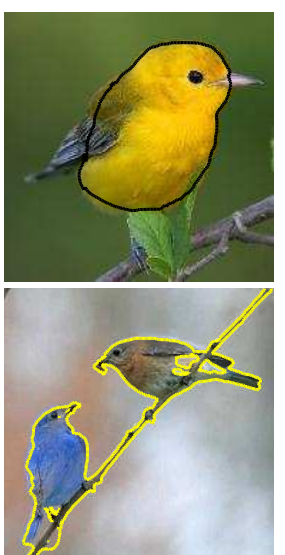

IAC model

Fig. 5. Real color images. Final curves of our model, vector-valued Chan-Vese model [10], and integrated active contour model (IAC).

$\{2,2.5,3,3.5\}, \theta_{\ell} \in\{0, \pi / 2\}, s_{\ell}=2$. Gabor based Chan-Vese model fails to detect the object on the top right side (even with $d=64$ filters with $\eta_{\ell} \in\{2,3,4,5\}$, $\left.\theta_{\ell} \in\{0, \pi / 4, \pi / 2,3 \pi / 4\}, s_{\ell} \in\{2,2 \sqrt{2}, 4,4 \sqrt{2}\}\right)$ because the intensity values of that object in Gabor transforms are very small compared with the ones of the other objects and close to the one of the background. But, our model detects all the objects well due to the local homogeneity.

In Fig. 7 , the images are composed of a background and an object with smoothly varying features. Here, we use $d=4$ filters with $\eta_{\ell} \in\{0.7,1.6\}, \theta_{\ell}=0$, $s_{\ell} \in\{4,4 \sqrt{2}\}$ in the first example, and $d=8$ filters with $\eta_{\ell} \in\{2,3,4,5\}, \theta_{\ell}=0$, $s_{\ell} \in\{4,4 \sqrt{2}\}$ in the second one. Due to a reason similar with the one in Fig. 1, Gabor based Chan-Vese model [11] fails to segment the actual object boundary, while our model detects it. For the IAC model, we use $p=2, G_{b_{1}}=1, G_{b_{2}}$ with $b_{2}=3.75$ (top), 0.5 (bottom) for the edge function. IAC model detects the object in the first example but not in the second one, which depends on the edge function $g(x)$. However, our model could segment the object in both cases without any prior work on the edge function like the IAC model.

\section{Conclusion}

In this article, we have proposed a novel non-local energy for image/texture segmentation. We have compared our active contour model with state of the art. We have illustrated the superiority of our model over the existing regionbased and/or edge-based active contour models. Due to the local homogeneity property, our segmentation model could detect regions with smoothly spatially varying features and segment several separated object with different features. Furthermore, our model is less sensitive to the choice of initial curves as well as to noise than edge-based active contour models. 


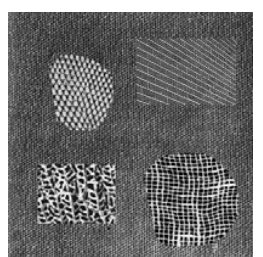

original

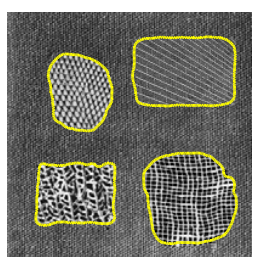

our model
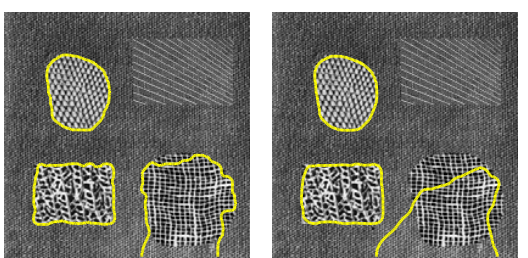

CV model with $d=8$ (left) and 64 (right)

Fig. 6. Texture segmentation with Gabor transforms. Comparison with Gabor based Chan-Vese model [11].

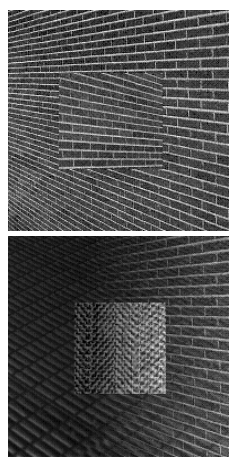

original

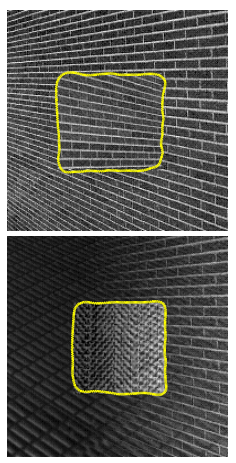

our model

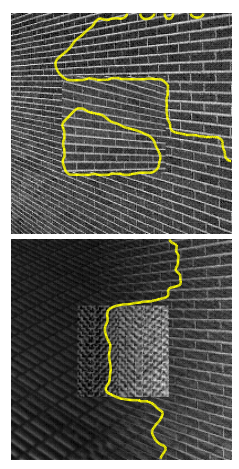

CV model

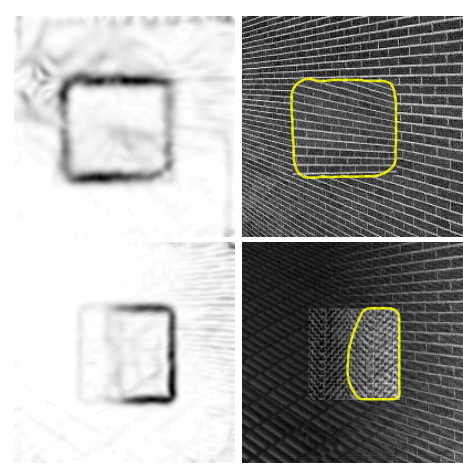

IAC model: edge function, final curve

Fig. 7. Texture segmentation with Gabor transforms. Image composed of a background and an object with smoothly varying features. Comparison with Gabor based ChanVese model [11] and IAC model [13].

\section{References}

1. Kass, M., Witkin, A., Terzopoulos, D.: Snakes: Active contour models. International Journal of Computer Vision 1 (1988) 321-331

2. Osher, S., Sethian, J.: Fronts propagating with curvature-dependent speed: Algorithms based on Hamilton-Jacobi formulations. Journal of Computational Physics 79 (1988) 12-49

3. Cohen, L.: On active contour models and balloons. CVGIP: Image Underst. 53 (1991) 211-218

4. Caselles, V., Catté, F., Coll, T., Dibos, F.: A geometric model for active contours in image processing. Numerische Mathematik 66 (1993) 1-31

5. Malladi, R., Sethian, J.A., Vemuri, B.C.: Shape modeling with front propagation: A level set approach. IEEE Trans. Patt. Anal. and Mach. Intell. 17 (1995) 158-175

6. Caselles, V., Kimmel, R., Sapiro, G.: Geodesic active contours. International Journal of Computer Vision 22 (1997) 61-79

7. Cohen, L.D.: Avoiding local minima for deformable curves in image analysis. Curves and Surfaces with Applications in CAGD, A. Le Méhauté, C. Rabut, and L. L. Schumaker (eds.), Vanderbilt University Press. (1997)

8. Mumford, D., Shah, J.: Optimal approximations by piecewise smooth functions and associated variational problems. Communications on Pure and Applied Mathematics XLII (1989) 
9. Chan, T., Vese, L.: Active contours without edges". IEEE Trans. Image Proc. 10 (2001) 266-277

10. Chan, T., Sandberg, B., Vese, L.: Active contours without edges for vector-valued images. J. Vis. Comm. Image Repr. 11 (2000) 130-141

11. Sandberg, B., Chan, T., Vese, L.: A level-set and Gabor based active contour algorithm for segmenting textured images. UCLA CAM Report 02-39 (2002)

12. Kimmel, R.: Fast edge integration. Geometric Level Set Methods in Imaging, Vision, and Graphics, S. Osher and N. Paragios, Eds. New York: Springer-Verlag (2003)

13. Sagiv, C., Sochen, N.A., Zeevi, Y.Y.: Integrated active contours for texture segmentation. IEEE Trans. Image Proc. 15 (2006) 1633-1646

14. Tsai, A., Yezzi, A., Willsky, A.S.: Curve evolution implementation of the mumfordshah functional for image segmentation, denoising, interpolation, and magnification. IEEE Trans. Image Proc. 10 (2001) 1169-1186

15. Li, C., Kao, C., Gore, J., Ding, Z.: Implicit active contours driven by local binary fitting energy. Proceedings of the CVPR'07 (2007) 1-7

16. Wang, X., Huang, D., Xu, H.: An efficient local chan-vese model for image segmentation. Pattern Recognition 43 (2010) 603-618

17. Lee, T.S., Mumford, D., Yuille, A.: Texture segmentation by minimizing vectorvalued energy functionals: The coupled-membrane model. In: Proc. ECCV '92. Volume 588. (1992)

18. Efros, A., Leung., T.: Texture synthesis by non-parametric sampling. IEEE International Conference on Computer Vision 2 (1999) 10-33

19. Efros, A., Freeman, W.: Image quilting for texture synthesis and transfer. Proceedings of SIGGRAPH (2001) 341-346

20. Buades, A., Coll, B., Morel, J.M.: A review of image denoising algorithms, with a new one. SIAM Mul. Model. and Simul. 4 (2005) 490-530

21. Kindermann, S., Osher, S., Jones, P.W.: Deblurring and denoising of images by nonlocal functionals. SIAM Mult. Model. and Simul. 4 (2005) 1091-1115

22. Gilboa, G., Osher, S.: Nonlocal operators with applications to image processing. SIAM Multiscale Modeling and Simulation 7 (2008) 1005-1028

23. Peyré, G., Bougleux, S., Cohen, L.: Non-local regularization of inverse problems. ECCV, Part III, LNCS 5304 (2008) 57-68

24. Gilboa, G., Osher, S.: Nonlocal linear image regularization and supervised segmentation. SIAM Mul. Model. and Simul. 6 (2007) 595-630

25. Elmoataz, A., Lezoray, O., Bougleux, S.: Nonlocal discrete regularization on weighted graphs: a framework for image and manifold processing. IEEE Trans Image Process. 17 (2008) 1047-1060

26. Houhou, N., Bresson, X., Szlam, A., Chan, T., Thiran, J.: Semi-supervised segmentation based on non-local continuous min-cut. In: Proc. SSVM '09, Springer (2009) 112-123

27. Bresson, X., Chan, T.: Non-local unsupervised variational image segmentation models. UCLA CAM Report 08-67 (2008)

28. Caldairou, B., Rousseau, F., Passat, N., Habas, P., Studholme, C., Heinrich, C.: A non-local fuzzy segmentation method: Application to brain mri. In: Proc. CAIP '09, Springer-Verlag (2009) 606-613

29. Shi, J., Malik, J.: Normlaized cuts and image segmentation. IEEE Trans. Patt. Anal. and Mach. Intell. 22 (2002) 888-905 